\section{Síndrome abdominal agudo debido a quiste infectado del mesenterio}

\begin{abstract}
RESUMEN
Los quistes del mesenterio son lesiones benignas intrabdominales que crecen a expensas de los vasos linfáticos del intestino; son más frecuentes en el mesenterio ileal y muy raros en la edad pediátrica. El síndrome abdominal agudo en la edad pediátrica es difícil de definir al ser un cuadro de origen múltiple y de presentación clínica muy variada; su síntoma principal es el dolor abdominal agudo, una de las causas que con mayor frecuencia motivan consulta en el Servicio de Urgencias Pediátricas. Los quistes de mesenterio son infrecuentes, se estima que se presentan en uno de cada 100000 ingresos por síndrome abdominal agudo en pediatría. El estudio de elección para el diagnóstico es el ultrasonido abdominal y el tratamiento óptimo es la resección completa del quiste. Se presenta el caso de una paciente con tumor abdominal ingresada para su estudio. Durante su evolución tuvo datos de irritación peritoneal por lo que se decidió realizar una laparotomía exploradora en la que se encontró un quiste del mesenterio, roto e infectado. Se efectuó hemicolectomía izquierda, lavado de cavidad y colostomía de una sola boca. A los tres meses de operada se hizo la restitución del tránsito intestinal con buenos resultados.
\end{abstract}

Palabras clave: síndrome abdominal agudo, quiste de mesenterio infectado, resección quirúrgica.

\section{Acute abdomen caused by an infected mesenteric cist}

\begin{abstract}
Mesenteric cysts are benign intra-abdominal lesions, which grow at the expense of the intestinal of the lymphatics, more frequent in the ileal mesentery and rare in children. The cause of an acute abdomen in children is hard to define as it is the clinical picture of multiple conditions; the main symptom is severe abdominal pain, one of the reasons most often source of consultation in the pediatric emergency department. Mesenteric cysts are unusual and are estimated to occur in one in 100,000 admissions for acute pediatric abdomen. The study of choice for the diagnosis is ultrasound (USG) abdominal. Optimal treatment is complete resection of the cyst. We present case of a girl with an abdominal tumor, admitted for study. The patient presents peritoneal irritation which required an exploratory laparotomy during which a rupture infected cyst was found in the mesentery. A left hemicolectomy was performed and the abdominal cavity was washed. A single neck colostomy was done. A three month after surgery intestinal normal transit was successfully performed.
\end{abstract}

Key words: acute abdomen, infected mesenteric cyst, surgical resection.
José Martín Palacios-Acosta ${ }^{1}$

Vanessa Carrasquel-Valecillos ${ }^{2}$

Angélica León-Hernández ${ }^{3}$

Jaén Echávez-del Riego ${ }^{2}$

Fabián Ricardo Castañeda-Bochm ${ }^{4}$

Daniel Hernández-Arrazola ${ }^{5}$

${ }^{1}$ Jefe de Servicio Cirugía Oncológica Pediátrica, Instituto Nacional de Pediatría.

${ }^{2}$ Médico residente de Cirugía Oncológica, Instituto Nacional de Pediatría.

${ }^{3}$ Médico General UNAM-FESI. Secretaria de Salud (CNEG y SR).

${ }^{4}$ Médico General, Hospital Ángeles Metropolitano.

${ }^{5}$ Médico adscrito de Cirugía Oncológica, Instituto Nacional de Pediatría.
Recibido: 19 de diciembre del 2013

Aceptado: 12 de marzo del 2015

Correspondencia: Dr. Daniel Hernández Arrazola Instituto Nacional de Pediatría

Av. Insurgentes Sur 3700-C

CP 04530 México, D.F.

Tel. 10840900 ext. 1322

Este artículo debe citarse como

Palacios-Acosta JM, Carrasquel-Valecillos V, LeónHernández A, Echávez-del Riego J, Castañeda-Bochm FR, Hernández-Arrazola D. Síndrome abdominal agudo debido a quiste infectado del mesenterio. Acta Pediatr Mex 2015;36:397-401. 


\section{INTRODUCCIÓN}

Los quistes de mesenterio son lesiones benignas intrabdominales que crecen a expensas de los vasos linfáticos del intestino; pueden localizarse desde el duodeno hasta el recto pero son más frecuentes en el mesenterio ileal y en el mesocolon; ${ }^{1,2}$ son raros en la edad pediátrica, con una incidencia de 1:27 000 casos con mayor frecuencia en el sexo femenino. ${ }^{3,4}$ La forma de presentación es variable, la manifestación clínica más frecuente es el dolor abdominal leve y difuso, aunque puede ser intenso, lo que depende del tamaño, la localización o de las posibles complicaciones como la obstrucción intestinal, la rotura, vólvulos, hemorragia o infección con formación de abscesos en los quistes, lo que generalmente se relaciona con las dimensiones del quiste. Puede palparse una masa aproximadamente en $75 \%$ de los casos. ${ }^{5,6}$

El estudio de elección para el diagnóstico es el ultrasonido abdominal; sólo si se requiere información adicional para confirmar el diagnóstico se realiza una tomografía con medio de contraste o una resonancia magnética nuclear. ${ }^{7,8}$

El tratamiento óptimo es la resección completa del quiste ya que puede haber recidiva cuando sólo se aspira su contenido y se reseca parcialmente. Se ha sugerido la marsupialización del quiste cuando no es posible su extirpación total, por la posibilidad de lesionar los órganos adyacentes. En ocasiones, el quiste comparte de manera íntima su irrigación con algún segmento de intestino, en cuyo caso se recomienda la resección intestinal del segmento involucrado. El tratamiento también puede realizarse con cirugía de mínima invasión. ${ }^{9,10}$

Se presenta el caso de una paciente con un tumor abdominal que fue ingresada para su estudio. Durante su evolución tuvo datos de irritación peritoneal y de respuesta inflamatoria que requirieron estabilización hemodinámica y, posteriormente, cirugía en la que se encontró un quiste de mesenterio roto e infectado.

\section{CASO CLÍNICO}

Niña de un año de edad que inició su padecimiento con dolor y distensión abdominal, fiebre de $38^{\circ} \mathrm{C}$, sin náuseas ni vómitos. En la exploración física se encontró irritable, con Ilanto, mucosa oral deshidratada. Se palpó el abdomen con evidencia de una masa no móvil, dolorosa, que abarcaba la fosa ilíaca izquierda, flanco izquierdo e hipogastrio. Al resto de la exploración sin alteraciones. Una radiografía abdominal mostró desplazamiento de las asas intestinales de izquierda hacia la derecha. Se realizó un ultrasonido que reveló un tumor de componentes mixtos dependiente del ovario, por lo que se envió a evaluación. Para completar el protocolo se decidió efectuar una tomografía abdominopélvica que mostró lesión quística de $15 \times 10 \times 14 \mathrm{~cm}$, multilobulada, con paredes de $3 \mathrm{~mm}$ de grosor que desplazaba al colon descendente y a las asas del intestino delgado (Figura 1). La paciente tuvo datos de respuesta inflamatoria que requirieron apoyo ventilatorio y el uso de aminas.

En la exploración física se apreció el abdomen blando, sin evidencia por palpación de la masa abdominal por la cual había ingresado. Esto hizo sospechar de la rotura del tumor y se decidió realizar una laparotomía exploradora. Se encontró una lesión multiquística rota a la cavidad abdominal (Figura 2) con material purulento de un quiste infectado y necrosado de mesenterio (Figura 3). Se tomó una muestra de líquido purulento. Se realizó una hemicolectomía izquierda por el compromiso vascular, lavado de la cavidad y colostomía de una sola boca. El diagnóstico de patología fue quiste de mesenterio roto e infectado, con necrosis de colon descendente y sigmoides sin evidencia de perforación. 


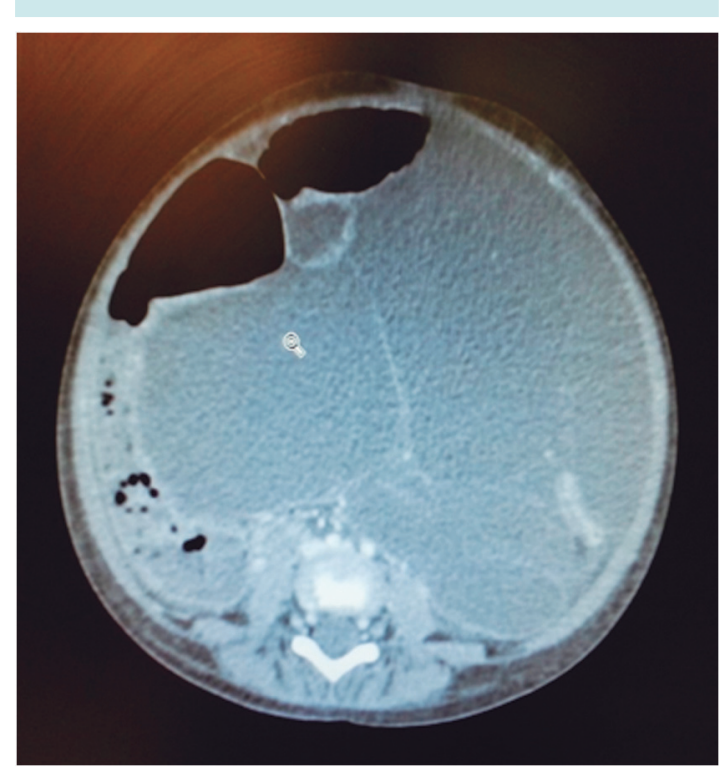

Figura 1. Tomografía abdominopélvica: lesión quística multilobulada.

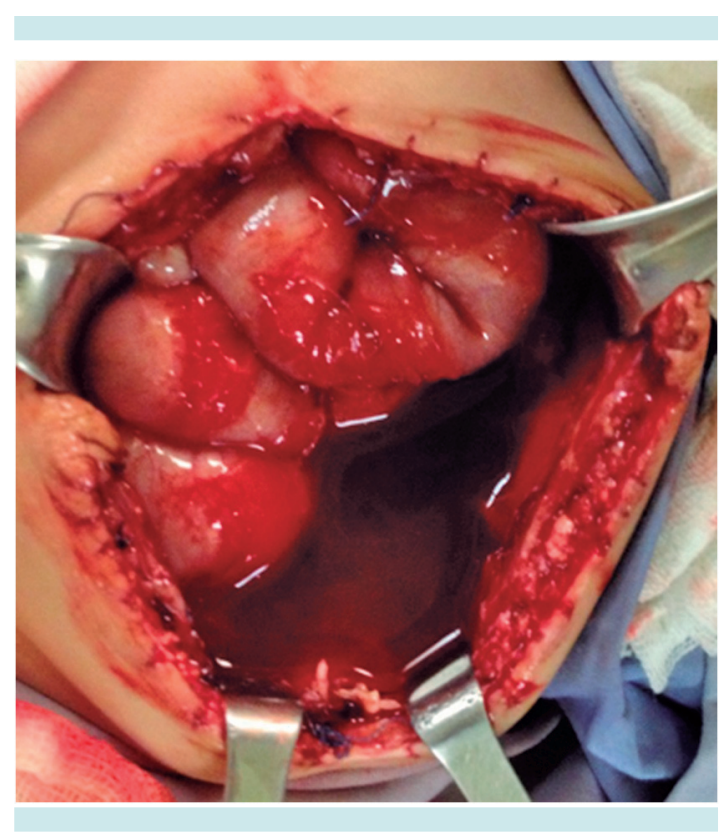

Figura 2. Lesión multiquística con rotura a la cavidad abdominal.

El posoperatorio fue favorable, sin complicaciones. A los tres meses de seguimiento se realizó

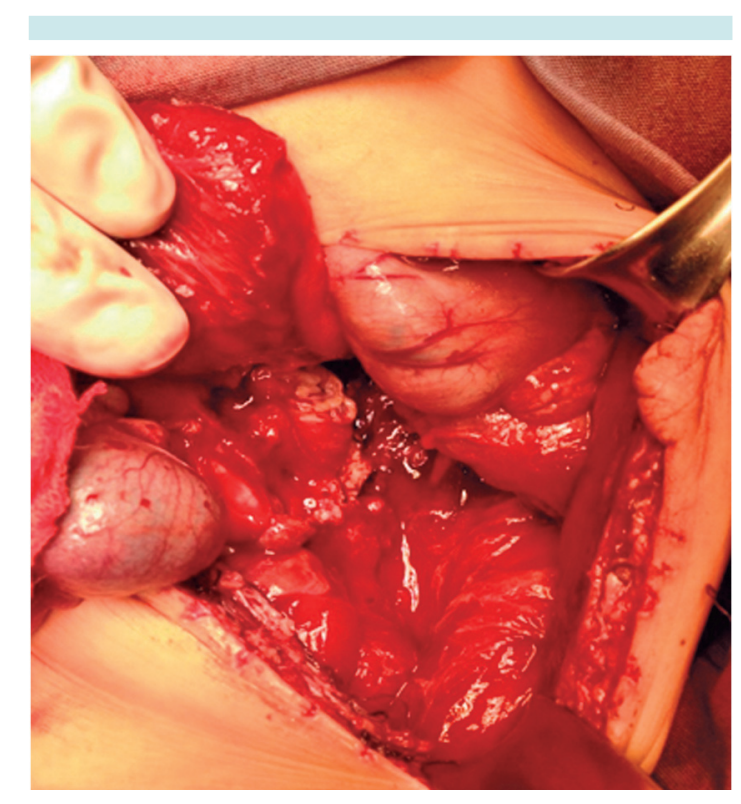

Figura 3. Quiste infectado del mesenterio.

la restitución del tránsito intestinal con buen resultado. Actualmente se encuentra en vigilancia con evolución satisfactoria y tránsito intestinal normal, sin recidiva a tres años.

\section{DISCUSIÓN}

Es difícil definir a que patología se debe el síndrome abdominal agudo en niños por ser un cuadro de distintos orígenes y de clínica muy variada. En general, se puede decir que el síntoma principal es el dolor abdominal agudo, uno de los motivos que con más frecuencia origina consultas en un Servicio de Urgencias Pediátricas. ${ }^{11}$ Los quistes de mesenterio son raros y se estima que se presentan en uno de cada 100000 ingresos por abdomen agudo en pediatría. ${ }^{12}$

Benevene, en 1507, informó el primer caso de un quiste de mesenterio en un niño de ocho años durante una autopsia. En 1842, Von Rokitansky documentó el primer caso de quiste de mesenterio quiloso. En 1880 se realizó la 
primera resección exitosa de un quiste de mesenterio. ${ }^{13,14}$

Los quistes de mesenterio provienen del saco linfático embrionario retroperitoneal situado en la región paravertebral adyacente a la raíz del mesenterio; están íntimamente relacionados con la formación del sistema venoso retroperitoneal. ${ }^{15}$ Varias teorías tratan de explicar el desarrollo de los quistes de epiplón y de mesenterio; entre ellas encontramos: 1) falla de los espacios linfáticos para unirse con el sistema venoso; 2) deficiencia de las uniones linfaticovenosas en el tejido perinodal; 3 ) falla en la unión de las hojas del mesenterio; 4) traumatismo oculto; 5) neoplasia y 6) degeneración localizada en los nódulos linfáticos. ${ }^{15}$

La teoría más aceptada es la de Gross, quien propone que se deben a una proliferación benigna de los linfáticos ectópicos en el mesenterio y que no tienen comunicación con el resto el sistema linfático. ${ }^{13,16}$

La clasificación de los quistes de mesenterio actual es la propuesta por Perrot y sus colaboradores ${ }^{17}$ basada en el origen de la lesión (Cuadro 1).

Las manifestaciones clínicas de los quistes de mesenterio dependen principalmente de la localización y del tamaño del tumor, así como de la relación que guarde con los órganos adyacentes. Se han establecido básicamente tres formas de presentación clínica: incidental, cuadro crónico y como síndrome de abdomen agudo. Se ha observado cierto número de síntomas que aparecen con frecuencia como el dolor abdominal, masa palpable, náusea, vómito y estreñimiento. ${ }^{18} \mathrm{El}$ dolor generalmente es difuso, con sensación de pesadez que se exacerba con los movimientos, fenómeno explicado por la tracción del mesenterio. Son frecuentes los periodos de obstrucción intestinal intermitente por compresión de las asas del intestino delgado o del colon. Puede aumen-
Cuadro 1. Clasificación de los quistes de mesenterio*

\begin{tabular}{ll}
\hline Quiste de origen linfático & $\begin{array}{l}\text { Quiste linfático simple } \\
\text { Linfangioma quístico } \\
\text { Quistes de origen mesotelial } \\
\text { Quiste mesotelial simple } \\
\text { Mesotelioma quístico } \\
\text { benigno } \\
\text { Mesotelioma quístico } \\
\text { maligno } \\
\text { Quistes entéricos de } \\
\text { duplicación intestinal } \\
\text { Quistes entéricos }\end{array}$ \\
$\begin{array}{ll}\text { Quistes de origen entérico de origen urogenital } \\
\text { Teratoma quístico maduro } \\
\text { (quiste dermoide) }\end{array}$ & $\begin{array}{l}\text { Seudoquistes no pancreáticos } \\
\text { De origen traumático } \\
\text { De origen infeccioso }\end{array}$ \\
*Según De Perrot y sus colaboradores. ${ }^{17}$
\end{tabular}

tar el perímetro abdominal y haber síntomas de vías urinarias por compresión vesical. Se ha descrito hemorragia rectal debida a hipertensión hemorroidal causada por la obstrucción venosa debida al tumor. ${ }^{12,19}$ La presentación clínica más frecuentemente señalada en la literatura especializada es un cuadro de síndrome de abdomen agudo debido a complicaciones del quiste como hemorragia, torsión, rotura, o bien a que produce lesión a órganos adyacentes que requiere de exploración quirúrgica. ${ }^{12}$

El método de diagnóstico de primera elección es el ultrasonido abdominal ${ }^{12,20}$ porque en las lesiones quísticas es más fácil identificar cámaras anecoicas y los septos, así como su relación con los órganos adyacentes. La tomografía abdominal permite definir el tamaño, la localización y, en ocasiones, la dependencia de la lesión. ${ }^{20,21}$

Existen múltiples diagnósticos diferenciales para esta afección, la entidad más frecuente con la cual debe realizarse el diagnóstico diferencial es el quiste de ovario, aunque deben investigarse otras lesiones intrabdominales como el seudoquiste del páncreas, la duplicación intestinal quística, el quiste de colédoco, el quiste des- 
Palacios-Acosta JM et al. Síndrome abdominal agudo debido a quiste

moide, el teratoma quístico, el quiste hidatídico o hidrocolecisto, los tumores retroperitoneales pediculados, la invaginación intestinal y la apendicitis complicada con absceso apendicular. ${ }^{15,22}$

El tratamiento de elección es la resección quirúrgica completa del quiste. La punción y drenaje del quiste expone a recidivas lo mismo que la resección parcial. No obstante, en los casos en los que no es posible su extirpación por la posibilidad de lesionar órganos adyacentes irresecables se ha sugerido la marsupialización del quiste. Cuando el quiste comparte íntimamente su irrigación con algún segmento de intestino se recomienda la resección intestinal del segmento involucrado. Se ha descrito el tratamiento laparoscópico en algunos casos. ${ }^{23-25}$

Nuestra recomendación para evitar recidivas y ante la evidencia de que esté involucrado el intestino es que se reseque completamente la lesión en bloque con el segmento de intestino comprometido. Tres meses después es factible realizar la restitución del tránsito intestinal sin problema como en el caso de nuestra paciente, quien a la fecha ha evolucionado satisfactoriamente con tránsito intestinal normal.

\section{REFERENCIAS}

1. Rivas ML, Asensio JA, Forno WJ, Petrone PM, Roldán G, García W. Quistes mesentéricos. Cir Gen 2002;24(1):61-5.

2. Saviano M, Fundaro S, Gelmini R, Begossi G, Perrone $S$, Farinetti $A$, et al. Mesenteric cystic neoformations: a report of two cases. Surg Today 2009;29:174-7.

3. Guerrero VJ. Quistes Mesentéricos. Web PEDiátrica 2005;3. http://www.webpediatrica.com/casosped/pdf/69_quistes_mesentericos.pdf

4. Sosa HR, Sánchez PC, Simón RL. Quiste del mesenterio: reporte de un caso y revisión de la literatura. Rev Cubana Cir 2007;46(4):1-9.

5. Bliss DP, Coffin CM, Bower RJ, Stockmann PT, Ternberg JL. Mesenteric cysts in children. Surgery 2004;115(4):571-7.

6. Pérez GR, Torres LE, Ruiz OJ. Quiste mesentérico: descripción de un caso. Cirujano General 2001;23(2):109-11.

7. Huis M, Balija M, Lez C, Szerda F, Stulhofer M. Mesenterio cyst. Acta Med Croatica 2002;56(3):119-24.
8. Luo CC, Huang CS, Chao HC. Intra-abdominal cystic lymphangiomas in infancy and childhood. Chang Gung Med J 2004;27(7):509-12.

9. Ricketts RR. Mesenteric and omental cysts. Pediatric Surgery 2008;5:1269-75.

10. Vialat SV, Labrada AE, Sandin HN. Quistes del mesenterio y epiplón en niños: Estudio de 20 años. Rev Cubana Pediatr 2004;76:3-5.

11. García AJ. Abdomen agudo en el niño. Protocolos diagnóstico-terapéuticos de Urgencias Pediátricas SEUP-AEP 2010;5:1-5.

12. Rosón RP, Fresno AA, Quintero BB. Quiste mesentérico retroperitoneal gigante con presentación como dispepsia. Gastroenterol Hepatol 2010;33(1):25-9.

13. Alvarado GR, Uribe VE, Fernández VS, Gallego GJ. Quistes de epiplón y mesenterio en niños. Acta Pediatr Méx 2001;22(5):332-36.

14. Martín ÁS, Chagolla SF, Quero HA, Vargas VM, Cisneros AR, Bulnes MD. Quistes de mesenterio y epiplón en niños. Estudio clínico de 21 casos. Pediatría de México 2099;11(1):20-3.

15. Guzmán VG. Quiste de mesenterio. Revisión. Rev Gastroenterol Mex 2013;68(3):235-38.

16. Sánchez FP, Mier DJ, Blanco BR, Martínez OJ. Quiste de mesenterio. Informe de un caso. Cir Ciruj 1999;67(4):143-5.

17. De Perrot M, Brudler M, Tostsch M, Mentha G, Morel P. Mesenteric cyst. Toward less confusion? Dig Surg 2000;17(4):323-28.

18. Rivera MA, Barrera YA, Basavilvazo RM, Hernández VM. Quiste mesentérico. Informe de un caso. Rev Med Inst Mex Seguro Soc 2008;46(4):423-26.

19. Jye-Y, Tan K, Chao S. Mesenteric Cyst: An institution experience over 4 years and review of the literature. World $J$ Surg 2009;33:1961-65.

20. Sato M, Ishida H, Konno K, Komatsuda T, Konno S, Watanabe $\mathrm{S}$, et al. Mesenteric cyst sonographic findings. Abdom Imaging 2000;25:306-10.

21. Phillips GW, Senapati AM, Young AE. Chylolymphatic mesenteric cyst: a diagnostic appearance on computed tomography. Br J Radiol 2008;61:413-15.

22. Takkal M, Ionescu G, Becker JH, Simson IW, Dreyer L. A complication of mesenteric lymphangioma: case report and brief review of literature. Acta Chir Belg 2006;96:130-2.

23. Cadeddu M, Mamazza J, Schlachta C, Seshadri P, Poulin E. Laparoscopic excision of retroperitoneal tumors: Technique and review of the laparoscopic experience. Surg Laparosc Endosc Percutan Tech 2001;11:144-7.

24. O'Brien MF, Winter DC, Lee G, Fitzgerald EJ, O'Sullivan GC. Mesenteric cysts: a series of six cases with a review of the literature. Ir J Med Sci 2009;168:233-6.

25. Morrison CP, Wemyss HS, Maddern GJ. A novel technique for the laparoscopic resection of mesenteric cysts. Surg Endosc 2002;16:219. 